\title{
240.
}

\section{NOTE ON A THEOREM IN SPHERICAL TRIGONOMETRY.}

[From the Philosophical Magazine, vol. xvII. (1859), p. 151.]

I AM not aware that the following theorem has been noticed: viz., in any spherical triangle, if as usual $a, b, c$ are the sides, and $A, B, C$ the opposite angles, then

$$
\begin{aligned}
& \sin b \sin c+\cos b \cos c \cos A=\sin B \sin C-\cos B \cos C \cos a, \\
& \sin c \sin a+\cos c \cos a \cos B=\sin C \sin A-\cos C \cos A \cos b, \\
& \sin a \sin b+\cos a \cos b \cos C=\sin A \sin B-\cos A \cos B \cos c .
\end{aligned}
$$

The demonstration is very simple; in fact we have

$$
\begin{aligned}
\sin b & \sin c+\cos b \cos c \cos A \\
& =\sin b \sin c\left(\sin ^{2} A+\cos ^{2} A\right)+\cos b \cos c \cos A \\
& =\sin b \sin c \sin ^{2} A+\cos A(\cos b \cos c+\sin b \sin c \cos A) \\
& =\sin B \sin C \sin ^{2} a+\cos A \cos a \\
& =\sin B \sin C\left(1-\cos ^{2} a\right)+\cos A \cos a \\
& =\sin B \sin C+\cos a(\cos A-\sin B \sin C \cos a) \\
& =\sin B \sin C-\cos B \cos C \cos a,
\end{aligned}
$$

which proves the theorem.

2, Stone Buildings, W.C., January 5, 1859.

A geometrical proof and interpretation are given, G. B. Airy, "Remarks on Mr Cayley's Trigonometrical Theorem, etc." Phil. Mag. same volume, p. 176. I transfer to this place the concluding sentence of the subsequent paper 243. "I take the opportunity of noticing that the theorem in spherical trigonometry, which I gave in the February Number, is not new, but, as pointed out by Prof. Chauvenet in the Mathematical Monthly (Cambridge, U.S.), is to be found in Cagnoli's 'Trigonometry' (1808)." 